\title{
Pseudochaotic behavior of deterministic automatic control systems of technical devices
}

\author{
Aleksandr Nikitin ${ }^{1 *}$, Konstantin Tagiltsev-Galeta ${ }^{1}$, Sergey Laktionov ${ }^{1}$, and Yonghui Yang $^{2}$ \\ ${ }^{1}$ Siberian State Industrial University, Novokuznetsk, 65400, Russia \\ ${ }^{2}$ University of Science and Technology Liaoning, Anshan, 14051, China
}

\begin{abstract}
A method for generating a pseudo-random response in a linear dynamic system is considered. An example of the system transition to pseudochaotic behavior is given. The calculation of fatigue loads in the toggle plate by the given method is presented. The advantage of this method in calculating fatigue loads is shown.
\end{abstract}

\section{Introduction}

In existing production systems, control processes are considered fully deterministic, but there are situations when fully deterministic systems exhibit chaotic behavior. Let us consider the control system in general (Fig. 1) [1].

The control object consists of a converter of the input signal $\mathbf{r}$ to the internal variable, a unit that compares the signal arriving via feedback and a unit for converting the internal variable to the output variable $\mathbf{y}$, as well as a feedback unit. Such a system is dynamic.

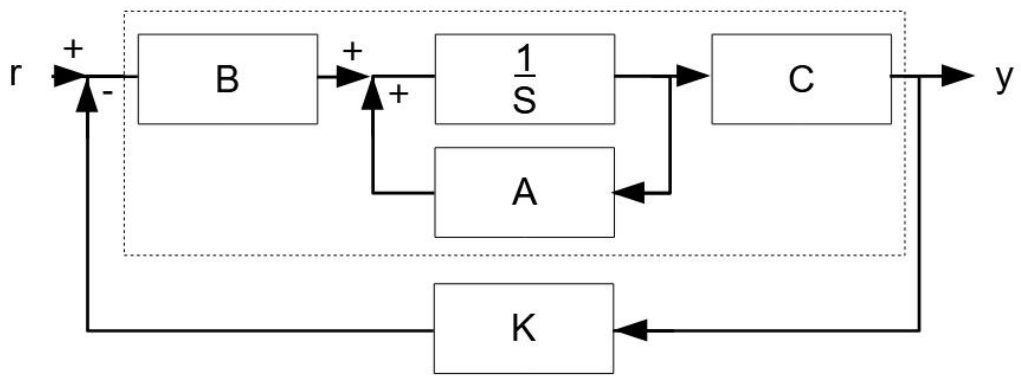

Fig. 1. Structure of the control system: $\mathbf{r}-$ initial action on the system, $\mathbf{A}$ - system state operator, $\mathbf{B}-$ transformation operator to an internal variable, $\mathbf{C}$ - transformation operator to the output variable, $\mathbf{1} / \mathbf{S}$ - differentiation operator, $\mathbf{K}$ - control operator, $\mathbf{y}$ - output variable.

The output of such a system is the curve shown in Fig 2.

\footnotetext{
${ }^{*}$ Corresponding author: nikitin1601@yandex.ru
} 


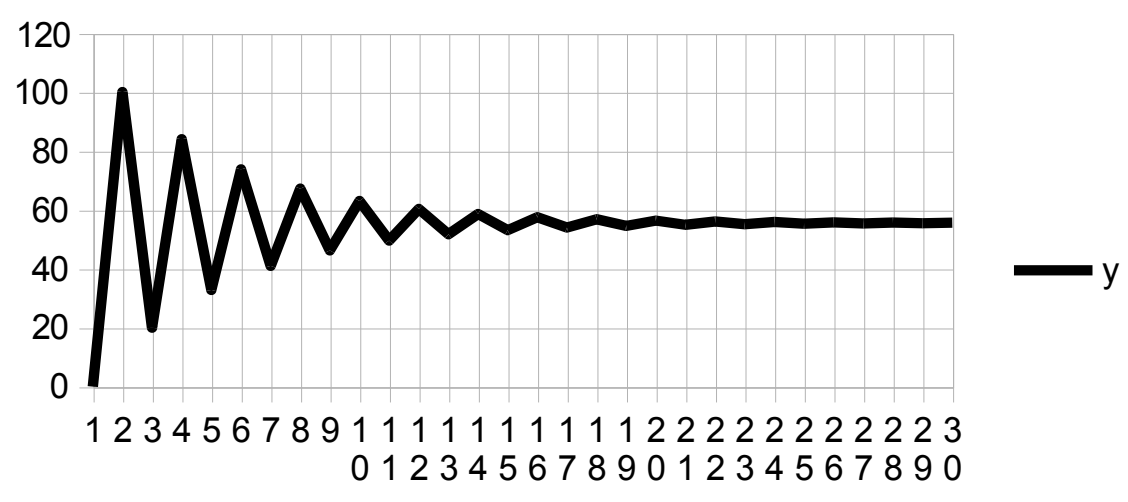

Fig. 2. Output signal of the control system.

\section{Method for generating a pseudo-random response in a linear dynamic system}

In modern information systems (including industrial ones), data conversion from one number system to another, which is linear, is most often used. For systems with a base $\mathbf{k}$, the number $\mathbf{x}$ is written as a sum of digits.

$$
x=\sum_{k=0}^{n-1} a_{k} b^{k}
$$

With a significant complexity of the algorithms used, methodological errors may occur, for example, the absence of a reverse transition to the original number system with base $\mathbf{k}$.

$$
\begin{gathered}
x=c_{k}=d_{l} \\
d_{l} \Rightarrow d_{k}
\end{gathered}
$$

Such an error may not be detected even during the preliminary testing phase, since the output values will not exceed the permissible values. However, the outputs received in this way will not be explicitly associated with the input values, generating pseudo-random outputs.

The resulting system has fully linear transformations, but is capable of generating a pseudo-random output signal (Fig. 3).

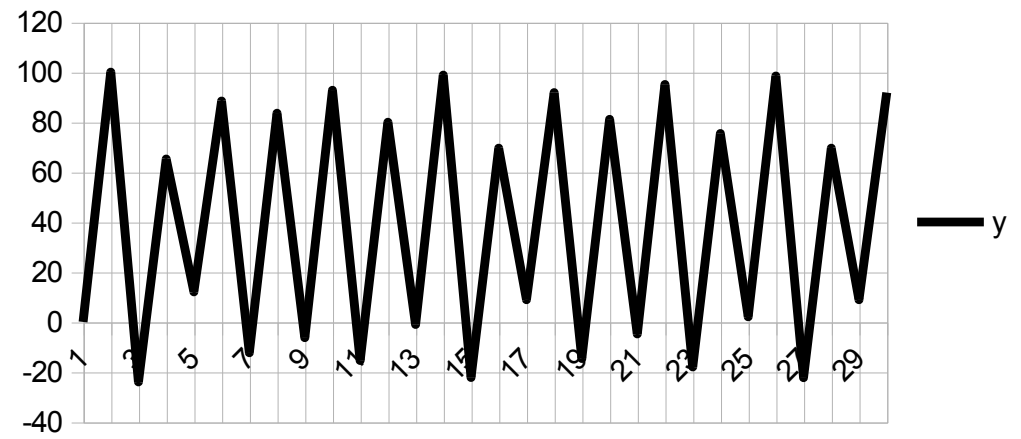

Fig. 3. Pseudo-random system output. 
The system exhibits random behavior similar to chaotic. Chaos in dynamical systems is usually caused by the nonlinearity of equations [2], but this system is capable of chaotic behavior with linearity of all equations included in it.

Let us consider the criteria for the system chaotic character [2]:

- divergence of trajectories according to Lyapunov;

- limited phase volume of the system;

- mixing of trajectories.

The resulting system demonstrates only some of these features:

- phase volume of the system is limited due to the presence of feedback;

- trajectories are mixed due to the limited phase space (Fig. 4).

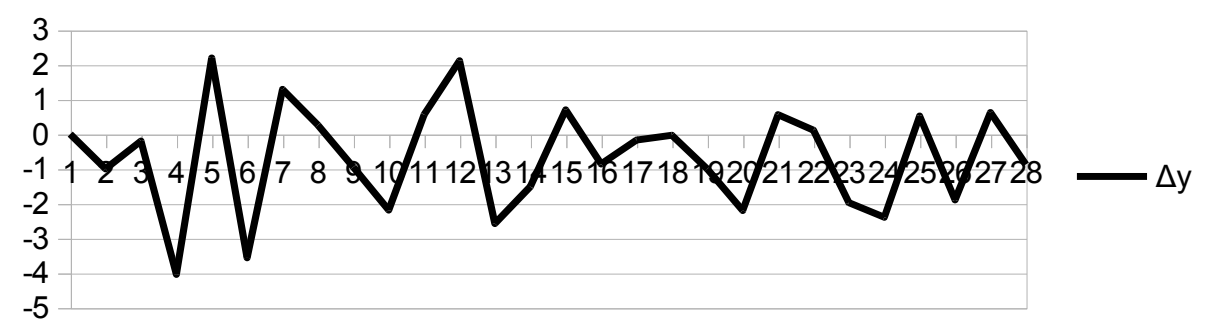

Fig. 4. Distance between close trajectories of the phase space.

The exponential divergence of trajectories according to Lyapunov is not expressed (Fig. 4), which does not allow judging about the chaotic nature of the resulting system. Nevertheless, the system obtained in this way makes it possible to study the behavior of objects under random influences.

One example of such a system is the simulation of rock crushing processes in a crushing machine. The crushing machine performs crushing of the rock, the characteristic of which is assumed to be unchanged ( $\sigma=$ const), while in real conditions in the crushing zone there may be objects with parameters exceeding the specified ones, which can lead to an emergency situation. As a preventive measure, various protective devices are used, for example, toggle plates. However, there is a possibility of false triggering of such devices when the material characteristics fluctuate within the permissible range. [3]

Methods of mathematical statistics are used to test the hypotheses of the destructibility of toggle plates from fatigue loads, but the results of such calculations give only an approximate estimate of the permissible load. [4]

Let us consider the process of fatigue loading of a toggle plates with a periodic load variable in magnitude.

$$
\frac{d l}{d N}=A * \Delta K^{n}
$$

where $l-$ crack length, mm; $\mathrm{N}-$ number of loads; A, $\mathrm{n}-$ empirical coefficients; $\Delta \mathrm{K}-$ range of the stress intensity factor.

Taking into account Maarten's research [5], the number of loads is calculated statistically:

$$
\lg (N)=\lg (\bar{N})+U * S,
$$

where $N$ is the average number of loads; $U$ is the quantile of the normal distribution law; $S$ is the variance of the service life. 
Obviously, the methods of fatigue theory only offer rough estimates. However, the proposed method makes it possible to simulate variable loads of different magnitudes in order to find a more accurate value of the fracture parameter of the spacer plate, in particular, to simulate the load signal of the spacer plate similarly to Fig. 3.

\section{Conclusion}

A method for generating dynamic systems with a random (pseudochaotic) output signal based on a linear system is proposed. It is suitable for modeling random processes and various emergency situations associated with non-stationarities, in particular, when calculating the number of loads on a toggle plates under subcritical loads.

\section{References}

1. B.T. Polyak, M.V. Khlebnikov, L.B. Rapoport, Mathematical theory of automatic control (2019)

2. S.D. Haitun, From an ergodic hypothesis to a fractal picture of the world: The birth and comprehension of a new paradigm (2007)

3. B.V. Klushantsev, A.I. Kosarev, Yu.A. Muizemnek, Crushers. Design, calculation of operating features (1990)

4. V.Z. Parton Fracture mechanics: From theory to practice (2016)

5. G.O. Johnston, Reliability Engineering, 3, 423-448 (1982) 